\title{
PREVALENCIA DE LESIONES CERVICALES PREMALIGNAS Y MALIGNAS
}

Liliana Mercedes Moreno, MD*, Arly Avila Agamez, MD*

Sonia Edith Saavedra Lancheros**, Katherine Zambrano Ochoa**.

\section{Resumen}

En el Hospital de San José desde diciembre de 2001 se viene realizando el control de calidad de las citologías cervicovaginales (CCV) del programa de Promoción y Prevención (P y P) de una EPS, que tiene por objetivo identificar en forma oportuna las lesiones precancerosas del cuello uterino. Este estudio se realizó para determinar la prevalencia de las lesiones premalignas y malignas en las CCV del programa entre enero de 2002 y diciembre de 2003. Se evaluaron 162.487 CCV, clasificadas según las categorías del sistema Bethesda, se determinó la prevalencia anual de citologías positivas y la prevalencia anual por cada categoría de lesión. Se encontró similitud de la prevalencia anual de cada lesión, lo que indica resultados satisfactorios para el programa de $\mathbf{P}$ y $\mathbf{P}$. Adicionalmente se realizaron comparaciones con cifras nacionales de la Liga de Lucha Contra el Cáncer y La Secretaría Distrital de Salud de Bogotá.

Palabras claves: citología cervicovaginal, programa de Promoción y Prevención, lesiones cervicales premalignas y malignas, sistema Bethesda, prevalencia.

\section{Introducción}

El cáncer cervicouterino es uno de los principales problemas de salud pública; en la actualidad en Colombia constituye la primera causa de muerte por cáncer en mujeres, con una incidencia aproximada de 44 casos por 100.000 habitantes. ${ }^{1}$ A nivel mundial, se presentan por año 500.000 casos nuevos y 200.000 muertes por esta misma causa. ${ }^{2}$ La gravedad de esta situación se acrecienta al reconocer que la enfermedad afecta a mujeres cada vez más jóvenes.

El virus del papiloma humano (VPH) constituye la principal causa para el desarrollo de cáncer de cuello uterino. Se han encontrado más de 75 tipos y cerca de 30 de ellos infectan el cérvix. Evidencias epidemiológicas muestran que al menos el 60\% de mujeres con vida sexual activa menores de 35 años se han contagiado. ${ }^{1}$

Residentes de III año servicio de Patología Hospital de San José. Fundación Universitaria de Ciencias de la Salud.

** Estudiantes de IV semestre de Citohistotecnología, Fundación Universitaria de Ciencias de la Salud, Hospital de San José

*** Este trabajo fue realizado como parte del Programa de Promoción y Prevención de una EPS en el Hospital de San José.Bogotá - Colombia. Enero 2002-diciembre 2003, bajo la tutoría de la Dra. Pilar Archila, Médica Patóloga Oncóloga del Hospital de San José y Profesora Asociada de la Fundación Universitaria de Ciencias de la Salud y de la Sra. Jeannette León Enciso, Instructora Asociada y Citohistotecnóloga, Fundación Universitaria de Ciencias de la Salud.
La técnica de la citología cervicovaginal implementada por el Papanicolaou (1941), es un método de tamización para identificar pacientes que podrían tener lesiones cervicales premalignas y malignas ocultas, contribuyendo a un diagnóstico definitivo mediante cuidadosas evaluaciones posteriores. Esta técnica de tamización es la base para el propósito que tienen los programas de P y P. La citología vaginal tiene varias cualidades, es fácil de realizar, aceptada por las pacientes, económica y certera.

La lectura y el reporte citológico han sufrido modificaciones a través del tiempo, desde Papanicolaou (1941) con el sistema de clasificación en cinco clases, Reagan (1953) con la clasificación en grados de displasia, Richard en grados de neoplasia intraepitelial cervical (NIC) y por último el sistema Bethesda $^{3}$ con las categorías de atípias escamosas de significado indeterminado (ASCUS), lesiones escamosas de bajo y alto grado (LIEBG y LIEAG), carcinoma escamocelular, atípias glandulares de significado indeterminado (AGUS), adenocarcinoma endocervical in situ y adenocarcinoma. 
En cumplimiento de las normas técnicas y administrativas del Ministerio de la Protección Social, según las resoluciones $412^{4}$ y $3384^{5}$ de 2000 , en Colombia se vienen incentivando las actividades de P y P dentro de las cuales está la detección temprana del cáncer de cuello uterino; las citadas resoluciones definen la norma técnica para la detección, atención y seguimiento de las pacientes, incluyen los reglamentos para los controles de calidad y el sistema de fortalecimiento de la gestión de las EPS y ARS.

Teniendo en cuenta lo anterior, en este estudio se evaluó mediante las prevalencias de la patología cervical, el comportamiento de las lesiones premalignas y malignas, en una población cautiva de bajo riesgo, así mismo se determinó el nivel en que se encuentra el programa de $\mathrm{P}$ y $\mathrm{P}$ de la EPS.

\section{Materiales y métodos}

Los datos recolectados se encuentran en el período comprendido entre enero de 2002 y diciembre de 2003, corresponden a los diagnósticos de las CCV del programa de $\mathrm{P}$ y $\mathrm{P}$ de una EPS, leídas en el Hospital de San José de la ciudad de Bogotá. Se obtuvo una población de $162.487 \mathrm{CCV}$ las cuales habían sido leídas en primera instancia por dos citólogas de la EPS y nueve citólogas del HSJ, posteriormente se revisó el diagnóstico efectuado por el grupo de seis patólogos del mismo hospital. Se determinaron las frecuencias de las lesiones premalignas y malignas a partir de un formato denominado control de calidad de la EPS diligenciado a diario por el patólogo.

También se utilizó un segundo formato denominado informe mensual de actividades de la EPS, donde se consolidaban la mayoría de los diagnósticos mensuales. Los datos restantes fueron recolectados y consolidados por las investigadoras. El método de procesamiento al que se sometieron los datos fue el registro de la información en una tabla en Microsoft Excel, que incluyó las citologías positivas categorizadas según Bethesda y las citologías negativas, mes a mes. Con los anteriores datos se determinó la prevalencia anual.
Después de examinar los datos y obtener los resultados, se compararon las cifras con estándares colombianos como la Secretaria Distrital de Salud de Bogotá y la Liga de Lucha contra el Cáncer. Además se compararon con la segunda edición The Bethesda System for Reporting Cervical Citology.

Diseño metodológico. Tipo de estudio: observacional -descriptivo: estudio de prevalencia. Población: CCV de una EPS del programa de P y P leídas en HSJ durante el período de enero de 2002 a diciembre de 2003. Muestra: en este proyecto se trabajó con toda la población.

\section{Crïterios de inclusión}

- Citologías cervicovaginales de una EPS del programa de P y P, leídas en el HSJ entre enero de 2002 a diciembre de 2003.

- Citologías cervicovaginales adecuadas (satisfactorias) para evaluación. Se aceptaron como adecuadas aquellas con presencia o ausencia de células endocervicales/componente de la zona de transformación y citologías con hemorragia e inflamación que dificultaban la evaluación hasta en no más del $75 \%$ de las células escamosas. ${ }^{3}$

\section{Criterios de exclusión}

- Citologías cervicovaginales inadecuadas (insatisfactorias) para evaluación. Se consideraron en este grupo aquellas cuyas láminas venían sin rotular, rotas, con defectos de fijación, extendidos gruesos o cuando se observaron menos del 10\% de células en el extendido. ${ }^{3}$

\section{Resultados}

Se obtuvo una población de 162.487 CCV durante los dos años de estudio. Para el 2002 la población con diagnósticos positivos correspondió a 1.811 casos, con una prevalencia de $2,35 \%$ y para el 2003, 1.808 casos con una prevalencia de $2,11 \%$, lo que indica una similitud en las frecuencias y en las prevalencias en el período analizado. 
Las prevalencias en el año 2002 fueron para ASCUS 1,202\% (926 casos), LIEBG 0,941\% (725 casos), LIEAG $0,153 \%$ (118 casos), carcinoma $0,012 \%$ ( 9 casos), AGUS 0,041\% (32 casos) y adenocarcinoma $0,001 \%$ (1 caso).

En el año 2003 el resultado fue para ASCUS 0,956\% (817casos), LIEBG 0,987\% (844 casos), LIEAG 0,091\% (78 casos), carcinoma 0,005\% (4 casos), AGUS 0,072\% (62 casos) y adenocarcinoma $0,003 \%$ (3 casos).

Dentro de las 162.487 CCV se analizó que la prevalencia anual de cáncer invasivo de cérvix, fue de $0,012 \%$ (9 casos) para el 2002 y $0,005 \%$ (4 casos), en 2003, es decir 13 pacientes en total.

Al evaluar las cifras del adenocarcinoma, 0,001\% ( 1 caso) para el 2002 y 0,003\% (3 casos) el 2003, se puede decir que aunque se presentaron cuatro casos en los dos años de este estudio, la prevalencia fue muy baja.

\section{Discusión}

La citología cervicovaginal es un método de tamizaje para la identificación precoz de lesiones premalignas y malignas del cuello uterino. Esta investigación muestra una similitud de la prevalencia de lesiones premalignas y malignas del cérvix durante los años 2002 y 2003, con una diferencia no relevante del $0,24 \%$, resultado que corresponde a tres citologías positivas más en el 2003, lo que indica que la variación por año de CCV positivas es baja. Es importante anotar que aunque la prevalencia anual de carcinoma invasor fue de $0,012 \%$ (9 casos) para 2002 y $0,005 \%$ (4 casos) el 2003, con un total de 13 casos, este estudio tiene la limitación de no poder definir cuales casos son incidentes para cada año, debido a que los datos consultados no diferencian entre nuevos y antiguos.

Por otra parte, el sistema Bethesda indica que las atipias escamosas de significado indeterminado (ASCUS) tienen una prevalencia menor del 5\%; al evaluar este aspecto se encontró que en el 2002 se presentó una prevalencia de 1,202\% (926 casos) y en el 2003 de $0,956 \%$ (817 casos), las cifras obtenidas en este estudio están por debajo de las esperadas. Así mismo, las atipias glandulares de significado indeterminado (AGUS) se presentan del 0,4 al 1\% según Bethesda. Analizando los resultados de este estudio se encontró un porcentaje de 0,041\% (32 casos) en el 2002 y $0,072 \%$ (62 casos) en el 2003. Lo anterior se podría explicar considerando que las pacientes de este estudio hacen parte de una población cautiva de bajo riesgo, proveniente de un programa de $\mathrm{P}$ y $\mathrm{P}$.

Las cifras de La Liga de Lucha Contra el Cáncer comparadas con esta investigación poseen una similitud importante. Podría ser que esta concordancia refleje que ambas poblaciones están sometidas a los mismos factores de riesgo para lesiones premalignas y malignas de cérvix. Además es posible que las pacientes de la Liga pertenezcan al grupo de atención básica primaria (Tabla 1).

Consultados los registros de La Secretaria Distrital de Salud de Bogotá del año 2003 y comparados con este estudio en el mismo período, mostraron una diferencia no relevante a pesar de que el número de la población del HSJ es menor. (Tabla 2). Con los anteriores resultados se podría concluir que la población de la EPS estudiada es representativa de las pacientes de los programas de $\mathrm{P}$ y $\mathrm{P}$ de la ciudad de Bogotá.

También se generan bastantes hipótesis, como sucede con la categoría de ASCUS que durante el 2002 arrojó un porcentaje de 1,202\% (926 casos), pero en el 2003 descendió la cifra a $0,956 \%$ (817 casos), lo que se cree favorable si se considera que la pequeña diferencia $(0,246 \%$ - 109 casos $)$ se debe a una clasificación diagnóstica más precisa en el 2003 de acuerdo con Bethesda.

Al finalizar esta investigación es necesario expresar sus limitaciones debido a que no se pueden generalizar los resultados, es decir no se puede extender al resto del país, teniendo en cuenta que la población de estudio es de bajo riesgo. 


\begin{tabular}{l|c|c|c|c}
\multirow{2}{*}{$\begin{array}{l}\text { Clasificación general de } \\
\text { las lesiones cervicales }\end{array}$} & \multicolumn{2}{c}{ CCV } & HSJ Comparación con la Liga de Lucha de contra el Cáncer & \multicolumn{2}{c}{ CCV Liga de Lucha Contra el Cáncer } \\
\cline { 2 - 5 } & 2002 & 2003 & 2002 & 2003 \\
\hline ASCUS & $1,202 \%(926)$ & $0,956 \%(817)$ & $* 1,98 \%$ & $* 2,11 \%$ \\
\hline AGUS & $0,041 \%(32)$ & $0,072 \%(62)$ & $(1,023)$ & $(925)$ \\
\hline LIEBG & $0,941 \%(725)$ & $0,987 \%(844)$ & $0,419 \%(216)$ & $0,535 \%(242)$ \\
\hline LIEAG & $0,153 \%(118)$ & $0,091 \%(78)$ & $0,250 \% /(129)$ & $0,327 \%(143)$ \\
\hline CARCINOMA & $0,012 \%(9)$ & $0,005 \%(4)$ & $0,126 \%(65)$ & $0,119 \%(52)$ \\
\hline ADENOCARCINOMA & $0,001 \%(1)$ & $0,003 \%(3)$ & $0,015 \%(8)$ & $0,032 \%(14)$ \\
\hline
\end{tabular}

Las cifras de la Secretaría presentan una categoría adicional denominada sospechoso de carcinoma, con una prevalencia de $0.097 \%$ (I8I casos), no susceptible de comparación con las cifras de este estudio.

\begin{tabular}{|c|c|c|}
\hline \multicolumn{3}{|c|}{ Comparación del año 2003} \\
\hline Lesiones cervicales & HSJ & $\begin{array}{c}\text { Secretaria } \\
\text { Distrital de Salud } \\
\text { de Bogotá }\end{array}$ \\
\hline ASCUS & $\begin{array}{c}0,956 \% \\
(817)\end{array}$ & $\begin{array}{l}2,839 \% \\
(5270)\end{array}$ \\
\hline AGUS & $\begin{array}{c}0,072 \% \\
(62)\end{array}$ & $\begin{array}{l}0,648 \% \\
(1203)\end{array}$ \\
\hline LIEBG & $\begin{array}{c}0,987 \% \\
(844)\end{array}$ & $\begin{array}{l}1,476 \% \\
(2740)\end{array}$ \\
\hline LIEAG & $\begin{array}{c}0,091 \% \\
(78)\end{array}$ & $\begin{array}{c}0,427 \% \\
(793)\end{array}$ \\
\hline CARCINOMA & $\begin{array}{c}0,005 \% \\
(4)\end{array}$ & $\begin{array}{c}0,054 \% \\
(101)\end{array}$ \\
\hline ADENOCARCINOMA & $\begin{array}{l}0,003 \% \\
\text { (3) }\end{array}$ & $\begin{array}{c}0,004 \% \\
(8)\end{array}$ \\
\hline
\end{tabular}

( ) - Número de casos. \% Prevalencia. Población Secretaría de Salud: 185.699 CCV (2003). Población HSJ; 85.485 CCV (2003).

\section{Conclusiones}

Este estudio determinó la prevalencia anual de las lesiones premalignas y malignas cervicales del programa de $\mathrm{P}$ y $\mathrm{P}$ de una EPS en el HSJ entre los años 2002- 2003, encontrando que de la población total de $162.487 \mathrm{CCV}$, el 2,35\% (1.811 casos) fueron positivas para algún tipo de lesión en el 2002 y para el año siguiente el 2,11\% (1.808 casos), estando estas cifras dentro de lo esperado para los programas de
P y $\mathrm{P}$ que esencialmente manejan pacientes de bajo riesgo. Se demostró similitud de la prevalencia de las lesiones en los dos años analizados, lo cual indica un comportamiento poco variable.

Por último, queremos resaltar que estudios como este permiten determinar el comportamiento de una entidad nosológica en un tipo de población determinado, lo que constituye un importante baluarte para analizar, monitorear, comparar y valorar el éxito de programas de P y P. en Salud.

\section{Referencias}

1. Cortes Yépes H. Papiloma virus y cáncer de cérvix. Rev Col Obst y Gin. 2002; 54 (2): 108.

2. Torres R, J S. Lesiones escamosas intraepiteliales cervicales. Rev Col de Obst y Gin 49 (4): p 217-223.

3. Salomon D, Nayar R ed. The Bethesda System for reporting cervical cytology: definitions, criteria, and explanatory notes. New York: Springer, p.1-20, 67-156.

4. COLOMBIA. MINISTERIO DE SALUD. Resolución N ${ }^{\circ}$ 00412 de febrero 25 de 2000 por la cual se establece las actividades, procedimientos e intervenciones de demanda inducida y obligatorio cumplimiento y adopta las normas técnicas y guías de atención para el desarrollo de las acciones de protección específica y detección temprana y la atención de enfermedades de interés en salud publica. Disponible en http://www.minproteccionsocial.gov.co/

5. COLOMBIA. MINISTERIO DE SALUD. Resolución $\mathrm{N}^{\circ}$ 3384 de diciembre 29 de 2000 por la cual se modifican parcialmente las resoluciones 412 y 1745 de 2000 y se deroga la resolución 1078 de 2000. Disponible en http: //www.minprotecciónsocial.gov.co/. 